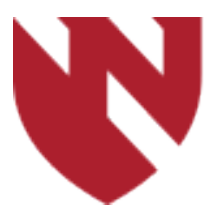

September 2020

\title{
Nontuberculosis Mycobacterium (NTM) Infection in Aquatic Workers with Purified Protein Derivative (PPD) and Gold QuantiFERON Tests
}

\author{
Lavanya Ravipati \\ University of Nebraska Medical Center \\ Sarah A. Howard \\ University of Nebraska Medical Center
}

Tell us how you used this information in this short survey.

Follow this and additional works at: https://digitalcommons.unmc.edu/gmerj

Part of the Higher Education Commons, and the Medicine and Health Sciences Commons

\section{Recommended Citation}

Ravipati, L., , Howard, S. A. Nontuberculosis Mycobacterium (NTM) Infection in Aquatic Workers with Purified Protein Derivative (PPD) and Gold QuantiFERON Tests. Graduate Medical Education Research Journal. 2020 Sep 29; 2(1).

https://digitalcommons.unmc.edu/gmerj/vol2/iss1/67

This Conference Proceeding is brought to you for free and open access by DigitalCommons@UNMC. It has been accepted for inclusion in Graduate Medical Education Research Journal by an authorized editor of DigitalCommons@UNMC.For more information, please contact digitalcommons@unmc.edu. 
Nontuberculosis Mycobacterium (NTM) Infection in Aquatic Workers with Purified Protein Derivative (PPD) and Gold QuantiFERON Tests

\section{Creative Commons License}

\section{(c) (1) (9)}

This work is licensed under a Creative Commons Attribution-Noncommercial-No Derivative Works 4.0 License. 
rates between the Lymph2Cx assay and the Hans and Choi algorithms were calculated.

Results: By the Lymph2Cx assay analysis, 36 cases (51\%) were classified as GCB, 25 cases $(36 \%)$ were classified as $\mathrm{ABC}$ and 9 cases $(13 \%)$ were intermediate/unclassified. The Hans algorithm classified $37 / 70$ cases $(53 \%)$ as GCB and $33 / 70$ cases $(47 \%)$ as nonGBC. The Choi algorithm classified 14/36 cases $(39 \%)$ as GCB and $22 / 36$ cases $(61 \%)$ as ABC. After excluding the 9 intermediate/ unclassified cases by the Lymph2Cx assay,
61 cases classified by the Hans algorithm and 30 cases classified by the Choi algorithm were compared to the Lymph2Cx. Overall, $54(89 \%)$ of cases classified by the Hans IHC algorithm and Lymph2Cx assay were concordant. 22 (77\%) classified by the Choi algorithm and Lymph2Cx assay were concordant.

Conclusion: The Lymph2Cx assay is a useful tool to determine COO in DLBCL.

https://doi.org/10.32873/unmc.dc.gmerj.2.1.064

\section{Nontuberculous Mycobacterium (NTM) Infection in Aquatic Workers With Purified Protein Derivative (PPD) and Gold QuantiFERON Tests \\ Lavanya Ravipati ${ }^{1}$, Sarah A. Howard ${ }^{1}$}

${ }^{1}$ University of Nebraska Medical Center, Clarkson Family Medicine

Mentor: Sarah A. Howard

Program: Clarkson Family Medicine

Type: Case Report

Background: Nontuberculous mycobacteria (NTM) infections are commonly caused by Mycobacterium marinum and fortuitum with M. marinum more likely to cause skin infections. Aquatic environments are a potential source of contact with M. marinum and fortuitum. Patients with NTM infections may present with positive tuberculin purified protein derivative (PPD) and/or gold QuantiFERON tests. However, PPD cannot differentiate between NTM infection and other tuberculous infections caused by different mycobacterial strains. We present an asymptomatic patient who presented for further evaluation of an off-site positive PPD and gold QuantiFERON Test. Methods: Patient informed consent was
obtained for this case report.

Results: A 31 year-old, healthy, female marine biologist employed in an aquatic environment presented to the clinic with a positive PPD and a positive gold QuantiFERON test. Her job exposes her to sea creatures that maybe infected with Mycobacterium marinum and fortuitum. She denied any history that was suggestive of tuberculous infection. A referral was made to specialist service for further evaluation at which time a repeat gold QuantiFERON test was performed and was noted to be positive. Following this work up, the patient, understanding her likelihood of having exposure to M. Marinum and fortuitum, preferred to follow a conservative treatment approach to monitor for any symptoms of active tuberculosis and seek follow up as needed.
Conclusion: Clinicians should not downplay the importance of aquatic occupations and hobbies in patients who exhibit positive PPD and gold QuantiFERON test. Healthcare providers should consider NTM infection in their differential diagnosis when examining patient populations exposed to aquarium water, saltwater, and freshwater environments. Preventative apparel like waterproof gloves can protect the exposed skin from infection. Clinicians can prevent NTM infections by increasing awareness and promoting prevention measures in at-risk populations, particularly since infection can be prevented with appropriate protective attire.

https://doi.org/10.32873/unmc.dc.gmerj.2.1.074

\section{Chemoembolization of Hepatocellular Carcinoma With Parasitic Blood Supply From the Inferior Phrenic Artery Lei Yu' ${ }^{1 *}$, Alex Hodson ${ }^{2 *}$, Timothy Donovan ${ }^{1}$, Christphor Vargo ${ }^{1}$ \\ 1 University of Nebraska Medical Center, Department of Radiology \\ ${ }^{2}$ University of Nebraska Medical Center, College of Medicine \\ *These authors contributed equally to this work.}

Mentor: Christopher Vargo

Program: Radiology

Type: Case Report

Background: Transarterial chemoembolization (TACE) is a commonly used embolotherapy for unresectable hepatocellular carcinoma (HCC). The hepatic artery is usually the vessel of choice, however, up to $30 \%$ of HCCs can develop an extrahepatic collateral (EHC) supply and failure to recognize $\mathrm{EHC}$ can lead to treatment failure. The most common extra hepatic artery supplying $\mathrm{HCC}$ is the inferior phrenic artery (IPA), with right IPA and left IPA comprising up to $83 \%$ and $12 \%$ of all collaterals respectively. Certain imaging features can help predict the presence of IPA collaterals and should be considered at the time of treatment.

Methods: We reviewed one recurrent HCC after radioembolization and one residual $\mathrm{HCC}$ after chemoembolization to identify tumor characteristics associated with the formation of EHC, especially IPA collaterals. Procedural considerations during IPA embolization and common complications are also reviewed.

Results: Several factors raise the suspicion for EHC development, including large size, location (segment VII), and prior arterial embolization. Avoiding non-target embolization is crucial in treatment planning as inferior phrenic artery supplies the diagram, and rarely, the pericardium and bowel. Prophylactic embolization to nontumor feeding branches can limit non-target embolization. 